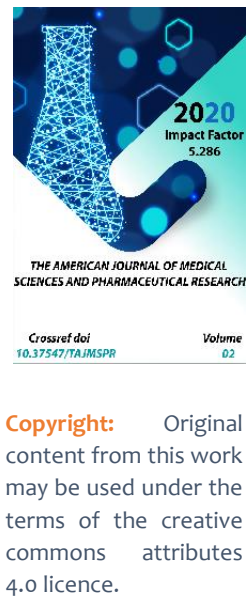

\title{
Results Of Surgical Treatment Of Recurrent Echinococcosis Of Lungs Depending On The Morphological Modifications
}

\author{
Bobonazarov Samariddin Dominovich \\ Pathological Anatomy With A Sectional Biopsy Course Samarkand State Medical Institute, \\ Samarkand City, Uzbekistan \\ Azamat Musakulovich Norjigitov \\ Pathological Anatomy With A Sectional Biopsy Course Samarkand State Medical Institute, \\ Samarkand City, Uzbekistan
}

\section{ABSTRACT}

Along with the increase in the number of new cases, there is a large percentage of echinococcosis relapses, the frequency of which, according to various authors, varies widely from 3.3 to $54 \%$, due to the existing terminology confusion. Material and methods of research. In the period from 2005 to 2019, 53 patients with recurrent echinococcosis of the lungs were operated on in the surgical Department of the 1st Sammi clinic. Research result. Immediate complications after surgery were observed in 6 (11.3\%) patients. Postoperative mortality was observed in 1 (1.9\%) case. The cause of death was acute cardiovascular failure. Conclusions. Analysis of the morphological characteristics of echinococcal cysts of the lungs showed that recurrent echinococcosis was most often found in echinococcus veterinorum (50.9\%), echinococcus hominis (37.7\%), and in echinococcus acephalocystis, there were no relapses of the disease, and if there were $(6-11.3 \%)$, we associate this with reinvasia of the parasite.

\section{KEYWORDS}

Echinococcosis of the lungs, morphological modifications, surgical treatment. 


\section{INTRODUCTION}

Human echinococcosis continues to be a severe parasitic disease. According to who and a number of other sources, it is widely spread and there is a significant increase in the incidence among the population [3, 7].

Along with the increase in the number of new cases, there is a large percentage of echinococcosis recurrences, the frequency of which, according to various authors, varies widely from 3.3 to $54 \%$, due to the existing terminology confusion $[1,4,5,6]$.

The aim of the study was to Find ways to optimize diagnostic and therapeutic measures in patients with recurrent lung echinococcosis, taking into account the morphological forms of echinococcal cysts.

\section{MATERIALS AND METHODS}

In the period from 2005 to 2019, 53 patients with recurrent echinococcosis of the lungs were operated on in the surgical Department of the 1st Sammi clinic. Echinococcosis of the right lung was detected in 29 patients, and left lung in 17 patients. In $76 \%$ of cases, the cysts were located in the lower lobe. Combined echinococcosis of both lungs was observed in 7 patients, the largest number of echinococcal cysts were also located in the lower lobe of both lungs. In recurrent echinococcosis of the lungs, solitary cysts were detected only in $24 \%$ of cases, mainly patients with multiple and combined echinococcosis prevailed in $76 \%$ of cases (table 1).

Table 1. distribution of patients by location of echinococcal cysts

\begin{tabular}{|c|c|c|c|}
\hline \multirow{2}{*}{ The nature and localization of the cysts } & \multicolumn{2}{c|}{ Number of patients } \\
\cline { 3 - 4 } & Right lung & 6 & 11,3 \\
\hline \multirow{2}{*}{ Solitary hydatid cyst } & Left lung & 7 & 13,2 \\
\cline { 2 - 4 } Multiple echinococcosis & Right lung & 23 & 43,4 \\
\cline { 2 - 4 } & Left lung & 10 & 18,9 \\
\hline Combined echinococcosis & 7 & 13,2 \\
\hline \multicolumn{2}{|c|}{ Total } & 53 & 100 \\
\hline
\end{tabular}

Depending on the size of the cyst, the patients were distributed as follows: small cysts were detected in 6 patients, medium cysts in 24 patients, large cysts in 17 patients, and in 6 patients cysts were gigantic in size over $20 \mathrm{~cm}$. Complicated cysts were observed in 15 patients, i.e. in $28 \%$ of cases. Among them, the cyst suppuration was in 7 patients, the cyst breakthrough in the bronchus was noted in 4 and in 3 patients the breakthrough was in the pleural cavity. In 1 case, there was copious hemoptysis. Studies were performed during preoperative preparation and in dynamics before normalization of indicators in the postoperative period. Among the instrumental 
research methods, radiography, computed tomography and ultrasound were used according to indications. We analyzed morphological modifications of echinococcal lung lesions. Modification of echinococcus hominis (figure 1) was observed in 20 (37.7\%) patients, echinococcus veterinorum (figure 2 )

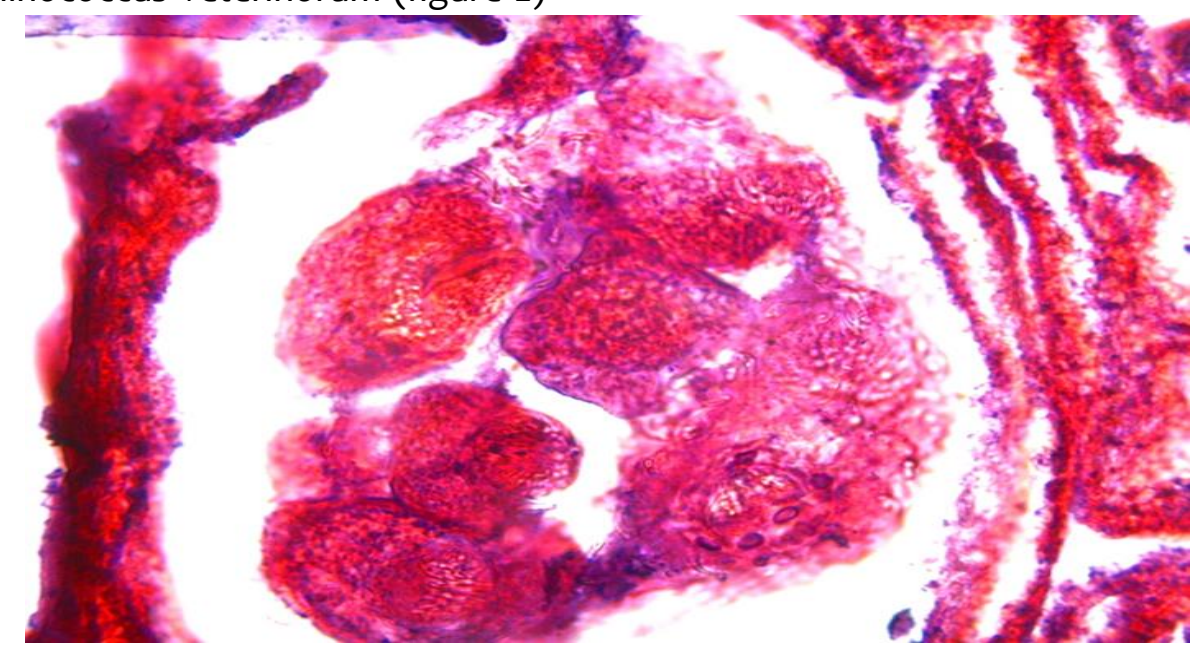

Fig. 1. Structure of an echinococcal cyst of the Echinococcus hominis modification.

Echinococcus scolex in the thickness of the fibrous capsule (color hematoxylin eosin, $x$ 400)

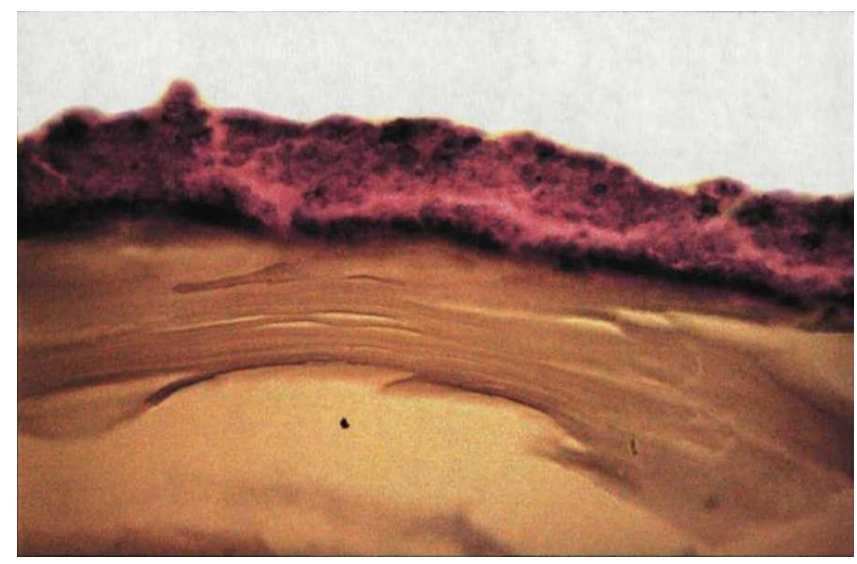

Fig. 2. Structure of echinococcal cyst modification echinococcus veterinorum (color hematoxylin eosin, $x$ 200) 


\section{DISCUSSIONS AND RESULTS}

The chest x-ray survey does not have any specific features characteristic of the modification of echinococcus hominis. The nature of the morphological form was finally judged intraoperatively. Modification of echinococcus hominis differs in that inside the cyst, in addition to the hydatid fluid, brood capsules with protoscolexes, there are daughter and sometimes grandchild bladders. Maternal cysts macroscopically have a matte rough surface and are colored milky white or whitish-yellow as shown on the slide. In this form, there is migration of scolexes beyond the chitinous shell into the thickness, or even beyond the fibrous capsule, and exogenous budding occurs with the growth of an echinococcal cyst. It was with this modification that relapses of the disease were observed at the site of pre-existing cysts. Preoperative diagnosis of Echinococcus veterinorum modification is possible using computed tomography of the chest. In this case, a specific feature is "hydatid sand" at the bottom of the cyst (Fig. 3).

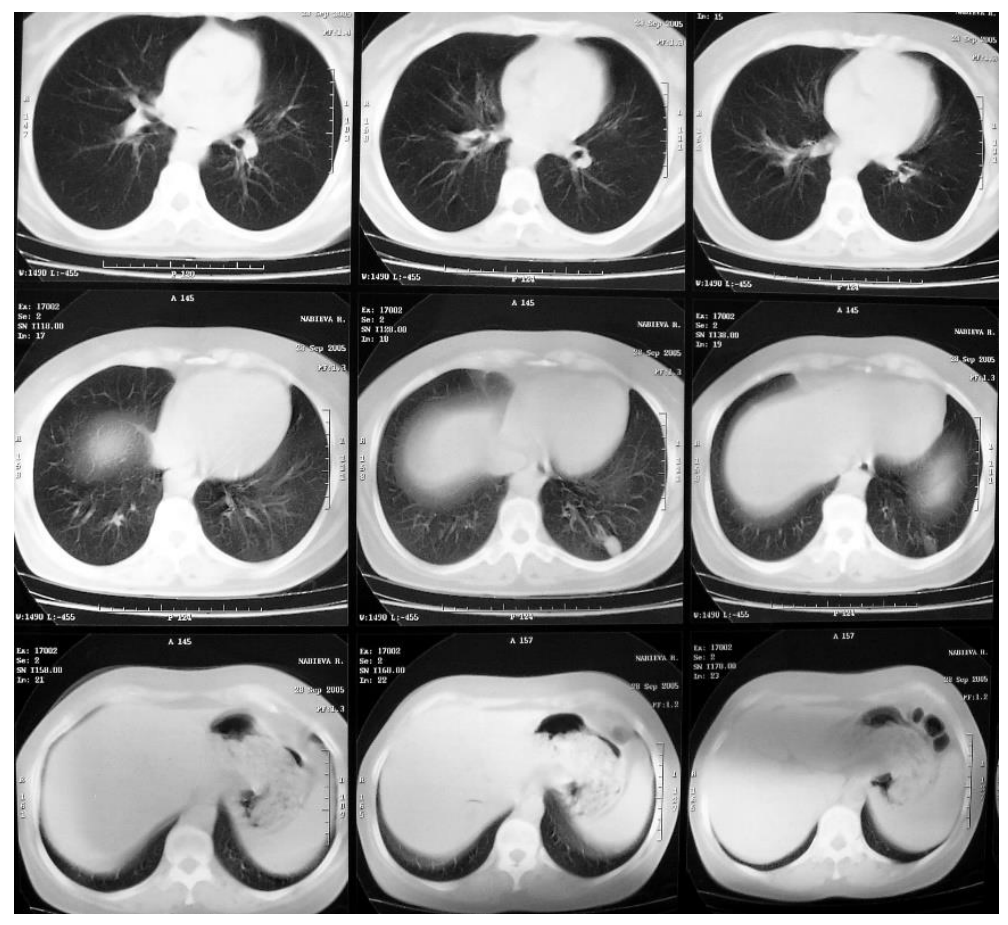

Fig. 3. Computed tomography of the chest "hydatid sand" for echinococcal cyst modification of echinococcus veterinorum

In the form of echinococcus veterinorum, there are only brood capsules and echinococcal fluid inside the lavrocysts. The formation of child bubbles does not occur. Echinococcus veterinorum is perhaps the most aggressive form of the parasite's existence, which is due to the high pressure of the hydatid fluid containing a large number of viable scolexes, which at the slightest violation of the integrity of the shell fall into the pleural cavity, causing massive damage to the internal organs with echinococcosis. According to our data, 
this form prevailed in the largest number, in $51 \%$ of cases.

Lavrocysts of the third modification of echinococcus acephalocystis (Fig. 4) were observed in 6 observations. These types of cysts are characterized by the absence of brood capsules and protoscolexes and are more common in animals than in humans.
Preoperative diagnosis of this modification of cysts is very difficult. X-ray and echographic characteristics of such cysts are very similar to non-parasitic lung cysts. Histological examination of the germinal membrane showed that its entire surface undergoes dystrophic changes, brood capsules are absent. Therefore, these cysts are not able to produce germ elements.

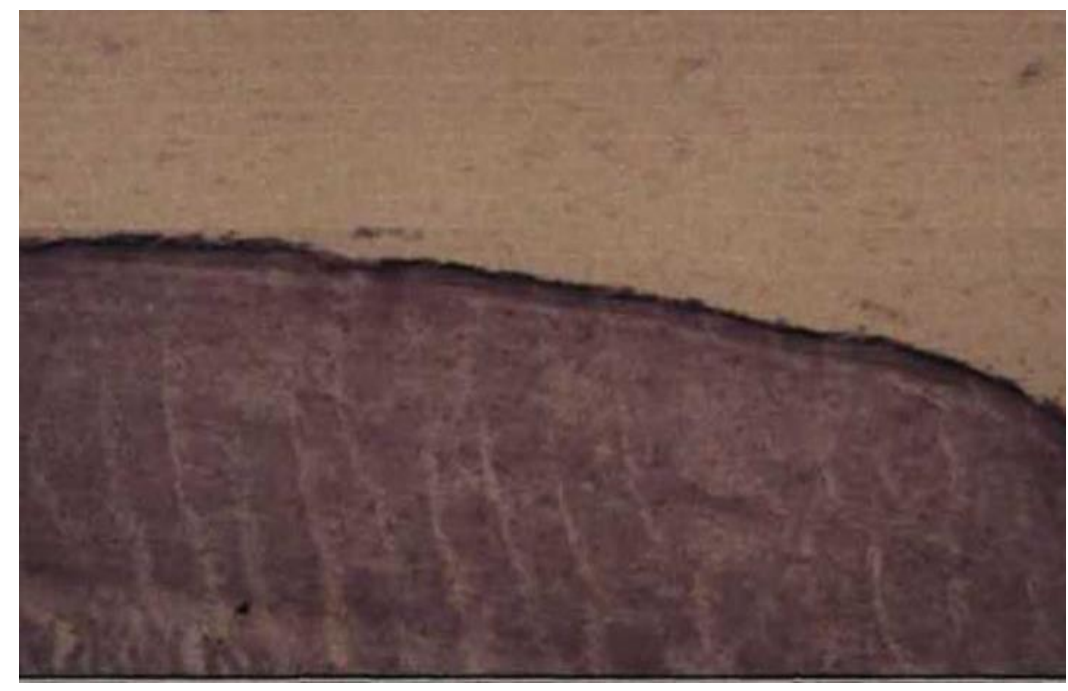

Fig. 4. Structure of an echinococcal cyst of the Echinococcus acephalocystis modification. (color hemotoxylin-eosin, x 200).

Research result. 53 patients with recurrent lung echinococcosis (100\%) underwent surgery. Complicated course of the disease was observed in 17 patients (32.1\%).

The timing of surgical interventions was determined by the nature of organ damage by the parasitic process, the presence of complications, the duration of their development, and concomitant pathology. Since most patients with recurrent echinococcosis were admitted to the hospital in a serious or moderate condition, with already developed complications from cysts, which required preliminary conservative treatment, surgical interventions were mainly delayed and planned.

To prevent intraoperative insemination, the pleural cavity was restricted with gauze swabs soaked in $100 \%$ glycerol solution. Then the cyst was punctured, its contents were evacuated, cystotomy and removal of the chitinous membrane with daughter and granddaughter bladders. A thick needle was used for the puncture, the end of which was attached to a syringe or to an electric suction. A 100\% glycerol solution was used to treat the residual cavity. 
The American Journal of Medical Sciences and Pharmaceutical Research (ISSN - 2689-1026)

In relation to the elimination of the residual cavity, the issue was resolved depending on the localization of the process, the size of the residual cavity, the presence of suppurative changes in the thickness of the fibrous capsule and surrounding tissues.

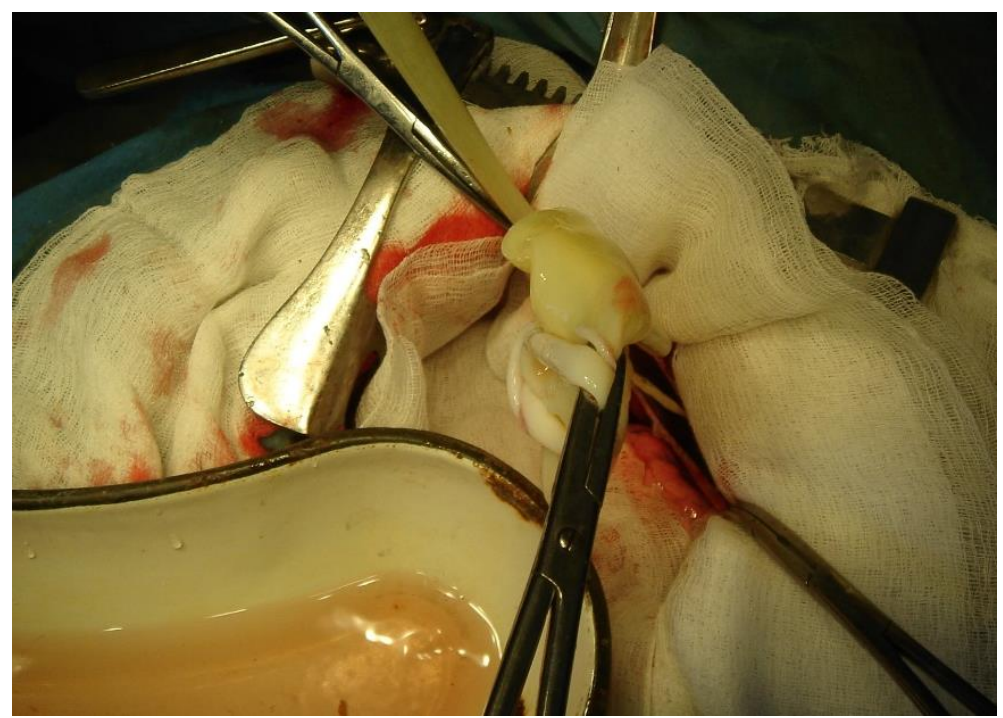

Fig. 5. Echinococcal cyst of the right lung segment VIII. Removal of the chitinous membrane

from the cyst lumen. The pleural cavity is limited by gauze swabs soaked in $100 \%$ glycerol

\section{solution}

mmediate complications after surgery were observed in $6(11.3 \%)$ patients. Postoperative mortality was observed in $1(1.9 \%)$ case. The cause of death was acute cardiovascular failure. Postoperative complications worsened the General condition of patients and prolonged the terms of their treatment and rehabilitation (table 2).

Table 2. Results of surgical treatment in the early postoperative period

\begin{tabular}{|c|c|c|}
\hline \multirow{2}{*}{ The nature of the complications } & \multicolumn{2}{|c|}{ Number of complications } \\
\cline { 2 - 3 } & a6c. & $\%$ \\
\hline Cardiopulmonary failure & $\begin{array}{c}1 \text { (lethal } \\
\text { outcome) }\end{array}$ & 1,9 \\
\hline Suppuration of the wound and empyema of the pleura & 1 & 1,9 \\
\hline Pneumonia and limited exudative pleurisy & 2 & 3,8 \\
\hline Summany pleurisy & 2 & 3,8 \\
\hline Total & 6 & $\mathbf{1 1 , 3}$ \\
\hline
\end{tabular}




\section{CONCLUSIONS}

There Are 3 morphological modifications of echinococcosis of the lungs: echinococcus veterinorum, echinococcus hominis and echinococcus acephalocystis, each of which has its own specific structure, which differs primarily in the structure of the germinal layer of the parasite.

Analysis of the morphological characteristics of echinococcal cysts of the lungs showed that recurrent echinococcosis was most often found in echinococcus veterinorum (50.9\%), echinococcus hominis (37.7\%), and in echinococcus acephalocystis, there were no relapses of the disease, and if there were (6 $11.3 \%)$, we associate this with reinvasia of the parasite.

The results of surgical treatment of echinococcal cysts of the lungs largely depend on a differentiated approach to choosing an adequate method of surgical treatment, taking into account the morphological form of the echinococcal cyst and observing the principles of aparasitarity and antiparasitarity.

\section{REFERENCES}

1. Karimov sh. I., Krotov N. F., Berkinov U. B. Possibilities of endovideosurgery in the treatment of lung echinococcosis //Bulletin of new medical technologies. - 2010. - Vol. 17. - no. 3.

2. Salimov sh. T. et al. The effectiveness of traditional and thoracoscopic lung echinococcectomy in children //Pediatric surgery. - 2012. - №. 4.

3. Chernousov A. F., Musaev G. H., Abarshalina M. V. Modern methods of surgical treatment of combined lung and liver echinococcosis / / Surgery. Magazine named after him. NO Pirogov. - 2012. - №. 7. - Pp. 12-17.
4. Mamatkulovna, V. A., \& Nurmamatovna, H. G. (2020). The Most Effective Drugs For Pecilomycose. //The American Journal of Medical Sciences and Pharmaceutical Research, 2(08), 64-68.

5. Shamsiev, A.m., Kurbaniyazov, Z. B., Shamsiev, Zh. A., Rakhmanov, K. E., \& Davlatov, S. S. (2017). Score in the choice of tactics for surgical treatment of liver echinococcosis. Problems of modern science and education, (37 (119)).

6. Sceuhangareeva R. Kh., Ishimov sh. S. Relapse of lung echinococcosis in children / / Surgery. Magazine named after him. NO Pirogov. - 2012. - №. 3. - Pp. 18-24.

7. Shevchenko, Yu. L., Nazirov, F. G., Ablitsov, Yu. A., Khudaibergenov, Sh. M., Musaev, G. Kh., Vasilashko, V. I., \& Ablitsov, A. Yu. (2016). Surgical treatment of echinococcosis of the lungs. Bulletin of the National medical and surgical center. $\mathrm{NI}$ Pirogova, 11 (3).

8. Ergashev N. sh., Pirnazarov B. T. Clinic, diagnostics and results of surgical treatment of children with complicated lung echinococcosis //post-graduate Doctor. - 2011. - Vol. 46. - No.3.3. - P. 404408. 